\title{
Magnetic-Field-Orientation Dependent Magnetoelectric Effect in FeBSiC/PZT/FeBSiC Composites
}

\author{
Jun-Xian Ye, ${ }^{1}$ Jia-Mian Hu, ${ }^{1}$ Zhan Shi, ${ }^{2}$ Zheng Li, ${ }^{1}$ Yang Shen, ${ }^{1}$ Jing Ma, ${ }^{1}$ and C. W. Nan \\ ${ }^{1}$ School of Materials Science and Engineering and State Key Lab of New Ceramics and Fine Processing, Tsinghua University, \\ Beijing 100084, China \\ ${ }^{2}$ Department of Materials Science and Engineering, College of Materials, Xiamen University, Xiamen 361005, China
}

Correspondence should be addressed to Jing Ma; ma-jing@tsinghua.edu.cn

Received 18 December 2013; Accepted 20 February 2014; Published 8 May 2014

Academic Editor: Tao Zhang

Copyright (C) 2014 Jun-Xian Ye et al. This is an open access article distributed under the Creative Commons Attribution License, which permits unrestricted use, distribution, and reproduction in any medium, provided the original work is properly cited.

\begin{abstract}
We investigate the magnetic-field-orientation dependent magnetoelectric (ME) effect in the $\mathrm{FeBSiC} / \mathrm{Pb}(\mathrm{Zr}, \mathrm{Ti}) \mathrm{O}_{3}(\mathrm{PZT}) / \mathrm{FeBSiC}$ laminates. It is shown that, by only using the bias-magnetic-field dependent ME response measured with the magnetic-field parallel to the surface plane of PZT slab, the magnetic-field-orientation dependent ME coefficient upon magnetic-fields of various amplitudes can be obtained via computer simulations. The simulation results match well the experimental measurements, demonstrating the applicability of the ME laminates-based sensors in detecting magnetic-fields with uncertain amplitudes and/or orientations in environment.
\end{abstract}

\section{Introduction}

The magnetoelectric (ME) effect is a phenomenon in which the electric polarization can be modified by a magnetic-field or a voltage output can be produced by applying a magneticfield on the materials, that is, direct ME effect, or, conversely, the magnetism is modified by an electric field [1-3]. It is well known that the ME effect in multiferroic ME composites is a product property of piezoelectricity and magnetostriction $[4,5]$, which could provide greater flexibility for applications as multifunctional devices due to the strong ME coupling at room temperature. Giant ME coupling has been achieved in various composites. For example, Dong et al. [6] obtained a remarkable ME coefficient of $22 \mathrm{~V} / \mathrm{cmOe}$ at a static magneticfield of 5 Oe with a collinear alternating magnetic-field of $6 \mathrm{Oe}(1 \mathrm{kHz})$ in a composite composed by a piezofiber/epoxy layer laminated between two layers of FeBSiC alloy. And Robert et al. [7] made great breakthrough that gets the extremely high ME coefficients of up to $9.7 \mathrm{kV} / \mathrm{cmOe}$ in air and $19 \mathrm{kV} / \mathrm{cmOe}$ under vacuum in thin-film composites.

From the application perspective, highly sensitive magnetic-field sensors can be obtained using the ME composites with high ME coefficients. Several prototypes of novel room temperature magnetic-field sensors [8-10] have been developed. Though the sensitivity is not as high as that of the superconducting quantum interference device (SQUID), the ME sensors show significant superiority in simple preparation and low cost as compared to the SQUID, which is sophisticated, expensive, energy consumptive, and only operated at cryogenic temperature. For instance, in a laminate of Terfenol-D/PZT/Terfenol-D, Zhai et al. [11] developed a novel ME sensor that can detect magnetic-fields down to pico-Tesla $\left(10^{-12} \mathrm{~T}\right)$ at room temperature, quite close to the $10^{-13} \mathrm{~T}$ in SQUID. Furthermore, the output bandwidth and frequency of the ME sensor can be improved by adding the number of ferromagnetic layers and/or using shapeengineered bimorph geometry $[12,13]$. More recently, Wang et al. [14] demonstrated an extremely low equivalent magnetic noise ME sensor based on both the giant ME effects in the Metglas/piezofiber heterostructure and a careful control of each internal noise source. However, for the application of magnetic-field sensor, besides the sensitivity, the influence of magnetic-field-orientation on the direct ME effect and the corresponding sensor design are also critically important issues regarding the uncertain, complex orientation distributions of magnetic-fields during practical operations. 


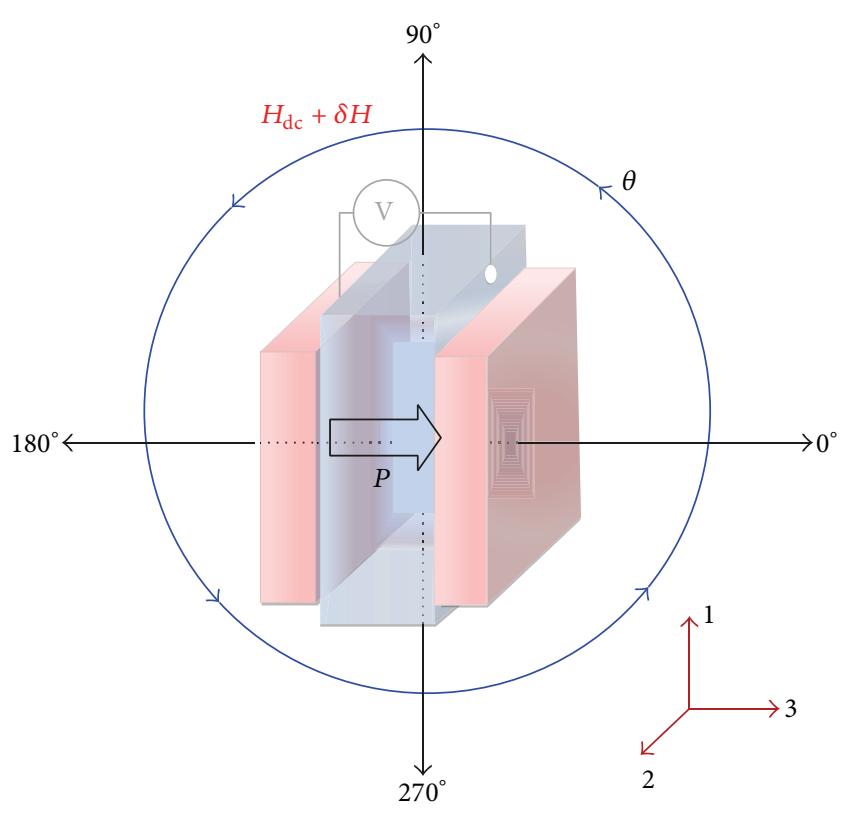

Figure 1: Schematic illustration of the $\mathrm{FeBSiC} / \mathrm{PZT} / \mathrm{FeBSiC}$ trilayer. The $\theta$ denotes the angle between the directions of magnetic-field and the electric polarization.

Previous studies have demonstrated that the measured ME coefficient varies with the change in angle between the poling field and applied magnetic-fields $[15,16]$. And it was also reported that ME response from a Metglas/PZT fiber laminates was strongly anisotropic, offering good sensitivity to magnetic-field variations only along its length direction, due to the giant permeability of Metglas [17]. In this work, we investigate the magnetic-field-orientation dependence of direct $\mathrm{ME}$ effect in a laminated $\mathrm{FeBSiC} / \mathrm{PZT} / \mathrm{FeBSiC}$ trilayer. It is found that the certain angle where the laminate gets its peak ME output directly depends on the external magneticfields. It is further demonstrated that, by using the magneticfield-dependent ME coefficients at one certain angle as the input, the ME response at other angles can be numerically calculated and agree well with the experimental results. Thus it would offer a simple and effective approach to determine the orientation of the magnetic-field in the applications of $\mathrm{ME}$ magnetic-field sensors.

\section{Experimental}

The simple ME trilayer made from two FeBSiC layers bonded on a PZT plate is schematically shown in Figure 1 . The PZT ceramic slab with dimensions of $10 \times 10 \times 0.5 \mathrm{~mm}^{3}$ (Sample 1) and $10 \times 5 \times 0.5 \mathrm{~mm}^{3}$ (Sample 2) was prepared via conventional solid-state processing and then polarized along the thickness direction (denoted by 3-axis) under a poling field of $3 \mathrm{kV} / \mathrm{mm}$, exhibiting a piezoelectric constant $d_{33}$ of $458 \mathrm{pC} / \mathrm{N}$ measured by a standard $d_{33}$ meter. The polarized PZT slab was bonded between two $30 \mu \mathrm{m}$ thick amorphous alloy FeBSiC layers (AT\&M Co., Ltd., Beijing) by epoxy. The amorphous alloy FeBSiC layer has a relatively high permeability $\mu_{r} \sim 230$ and saturation magnetostriction of $\lambda \sim 20$ ppm at $H_{\mathrm{dc}} \sim 100 \mathrm{Oe}$. The high $\mu_{r}$ of FeBSiC results in a small demagnetization field in the surface plane, enabling a high effective piezomagnetic coefficient at low biases ( $70 \mathrm{Oe}$ ), which made the laminate exhibit a large ME response at quite low $H_{\mathrm{dc}}$. But contrastively the thickness direction was the hard axis because of its large demagnetization field.

Room-temperature ME measurements are carried out by superimposing an alternating current (AC) magnetic-field $\delta H$ provided by a pair of Helmholtz coils driven by an arbitrary waveform generator (Agilent 33220A) onto a static magnetic-field $H_{\mathrm{dc}} \cdot H_{\mathrm{dc}}$ and $\delta H$ are parallel and fixed in the same direction, with the magnitude detected by a Gauss magnetometer (Lake Shore 455 DSP) in real time. During measurement, samples are rotated along axis 2 , and $\theta$ denotes the angle between the external magnetic-field and the electric polarization (Figure 1).

\section{Results and Discussion}

The dependence of the ME voltage coefficient $\alpha_{E}$ of Sample 1 on the static magnetic-field $H_{\mathrm{dc}}$ is present in Figure 2(a), with $\theta$ being fixed at $0^{\circ}$ or $90^{\circ}$; that is, the magnetic-field is perpendicular (out-of-plane) or parallel (in-plane) to the surface plane. It can be seen that, at $\theta=90^{\circ}, \alpha_{E}(=$ $\alpha_{E 31}$ herein; see Figure 1) increases almost linearly before one certain $H_{\text {dc-optimal }}$, where $\alpha_{E 31}$ reaches a maximum of $138 \mathrm{mV} / \mathrm{cmOe}$ (Point B) and then decreases nonlinearly as $H_{\mathrm{dc}}$ further increases. Such a variation trend should essentially be determined by the bias-dependent piezomagnetic coefficient $d \lambda / d H_{1}$ of the $\mathrm{FeBSiC}$ ribbon as reported in similar $\mathrm{CoFe}_{2} \mathrm{O}_{4} / \mathrm{PZT}$ composites [18], clearly demonstrating a strain-mediated direct ME effect. However, at $\theta=0^{\circ}$, the magnetostrictive deformation under $H_{\mathrm{dc}}$ along the 3-axis is negligible because of the strongly magnetic anisotropy of the Metglas FeBSiC. As a result, the $\alpha_{E}\left(=\alpha_{E 33}\right)$ is insignificant and shows little dependence on $H_{\mathrm{dc}}$ (Figure 2(a)). Such distinct angular anisotropy of the $\mathrm{ME}$ response can be used as an important criterion to determine the orientation of the external magnetic-field. However, in practical terms, we cannot simply identify the azimuth angle $\theta$ with the maximal ME output as the field-orientation parallel to the PZT surface.

For illustration, we further measure the $\alpha_{E}$ as a function of $\theta$ under various $H_{\mathrm{dc}}$; representatively, Points $\mathrm{A}, \mathrm{B}$, and $\mathrm{C}$ in Figure 2(a) are selected. Point $\mathrm{B}$ respects the certain $H_{\text {dc-optimal }}$ (73.2 Oe for Sample 1) where $\alpha_{E 31}$ reaches its maximum value, while Points $\mathrm{A}$ and $\mathrm{C}$ denote the magneticfield less and larger than $H_{\text {dc-optimal }}$. As seen in Figure 2(b), when $H_{\mathrm{dc}}$ is fixed at $36.6 \mathrm{Oe}$ (Point $\mathrm{A}$ in Figure 2(a)) and 73.2 Oe, the orientation dependence of ME coefficient shows a well-defined twofold symmetry in the corresponding polar plots, with the $\alpha_{E}$ reaching its maximal at $\theta=90^{\circ}$ and $270^{\circ}$ (i.e., the magnetic-field parallel to the surface plane) and almost vanishing at $\theta=0^{\circ}$ and $180^{\circ}$ (i.e., the magneticfield perpendicular to the surface plane). And the magnitude of the maximal $\alpha_{E}$ also increases with increasing $H_{\mathrm{dc}}$ from 36.6 Oe (Point A) to 73.2 Oe (Point B), following the trend in Figure 2(a). Of interest, however, the maximal $\alpha_{E}$ emerges 


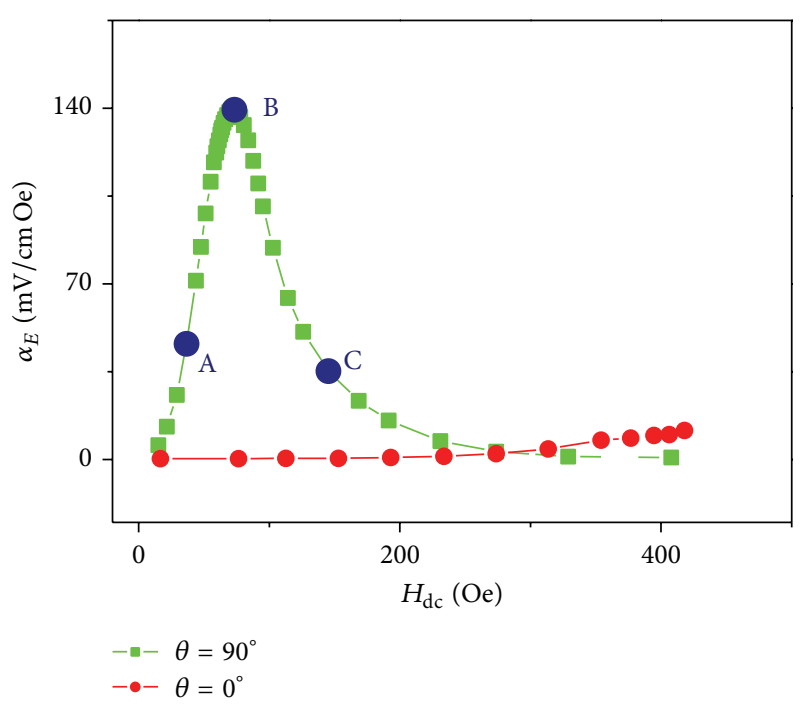

(a)

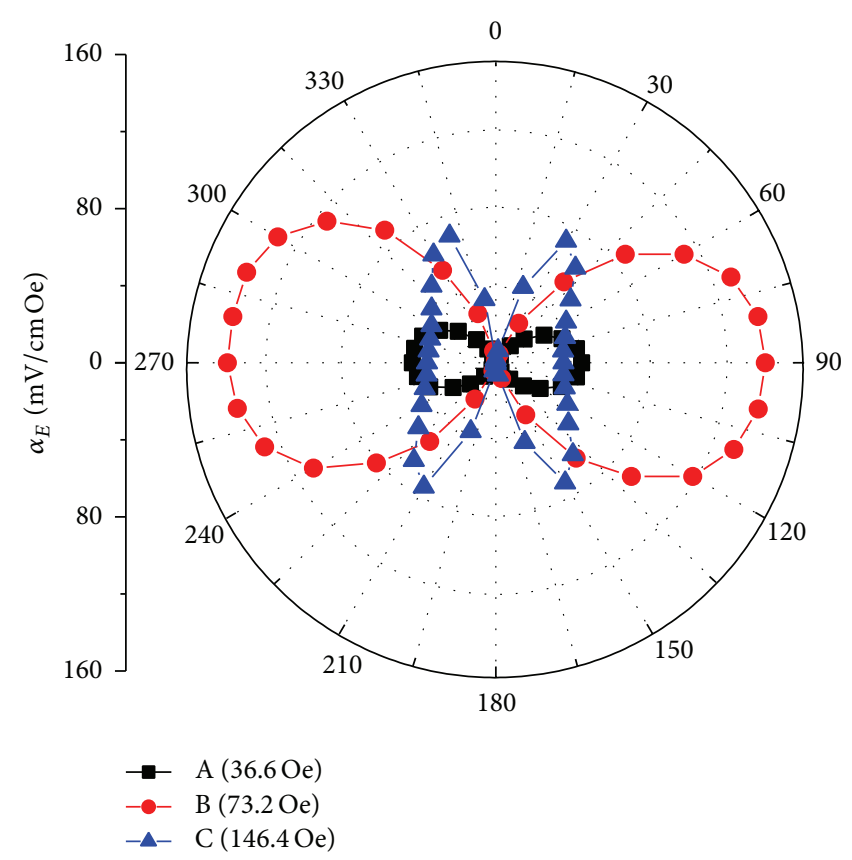

(b)

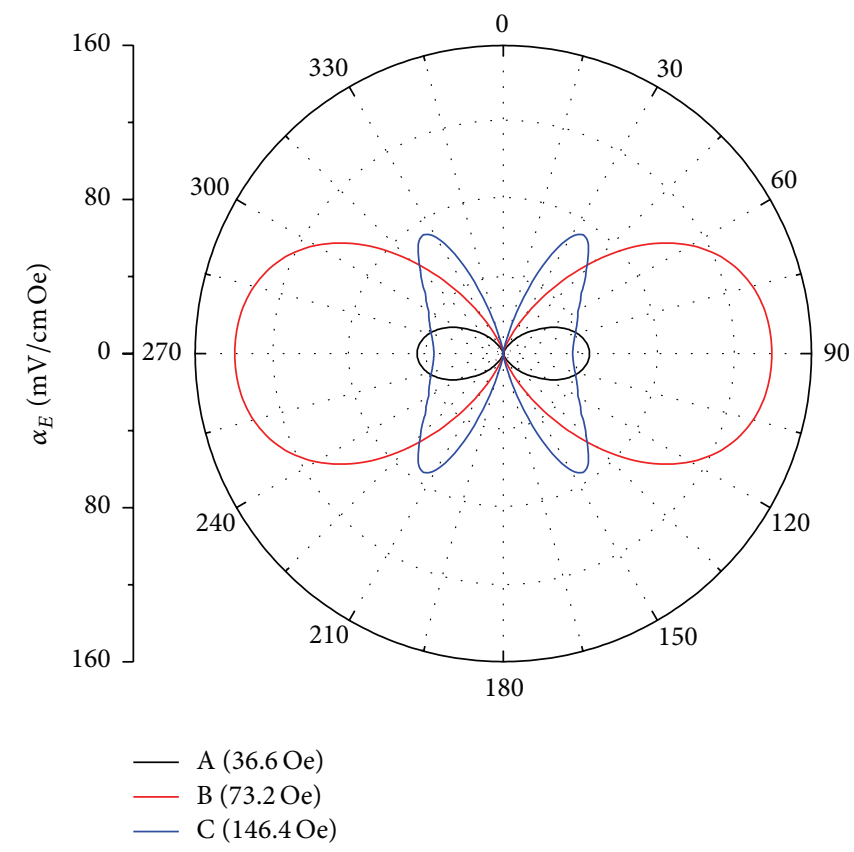

(c)

Figure 2: (a) Magnetic-field dependence of ME coefficient $\alpha_{E}$ in the FeBSiC/PZT/FeBSiC trilayer with a dimension of $10 \times 10 \times 0.5 \mathrm{~mm}^{3}$ for the PZT slab (Sample 1). (b) Experimentally measured polar plots of the angular dependent ME coefficient $\alpha_{E}$ in Sample 1 and (c) the computer simulation.

at $\theta_{\max } \approx 30^{\circ} / 210^{\circ}$ and $150^{\circ} / 330^{\circ}$ when $H_{\mathrm{dc}}$ reaches $146.4 \mathrm{Oe}$ (Point C in Figure 2(a)), showing a fourfold symmetry in its polar plot accordingly. Since only the magnetic-field parallel to the PZT surface would induce an obvious ME output and the ME coefficient exhibits magnetic-field dependence as shown in Figure 2(a), the effective magnetic-field $H_{\text {dc-eff }}$ of an arbitrary $H_{\mathrm{dc}}$ that induces a ME output can be expressed by
$H_{\text {dc-eff }}=H_{\mathrm{dc}} \times|\sin \theta|$. Consequently, the azimuth angles with maximum $\alpha_{E}$ upon an arbitrary $H_{\mathrm{dc}}$, denoted by $\theta_{\max }$, can be straightforwardly calculated by $H_{\text {dc-optimal }}=H_{\mathrm{dc}}\left|\sin \left(\theta_{\max }\right)\right|$. In this case, $H_{\mathrm{dc}}$ reaches $146.4 \mathrm{Oe}$ (Point $\mathrm{C}$ ) and $H_{\text {dc-optimal }}$ is 73.2 Oe for this sample, so $\left|\sin \left(\theta_{\text {max }}\right)\right|=H_{\text {dc-optimal }} / H_{\mathrm{dc}}=0.5$, and $\theta_{\max }$ can be $30^{\circ}, 210^{\circ}, 150^{\circ}$, and $330^{\circ}$, exhibiting a fourfold symmetry in its polar plot. 


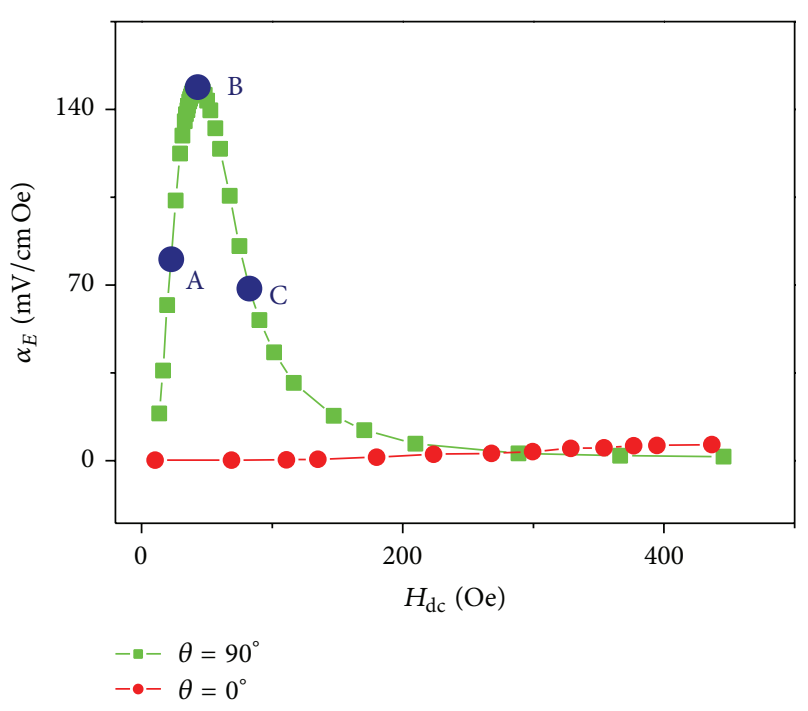

(a)

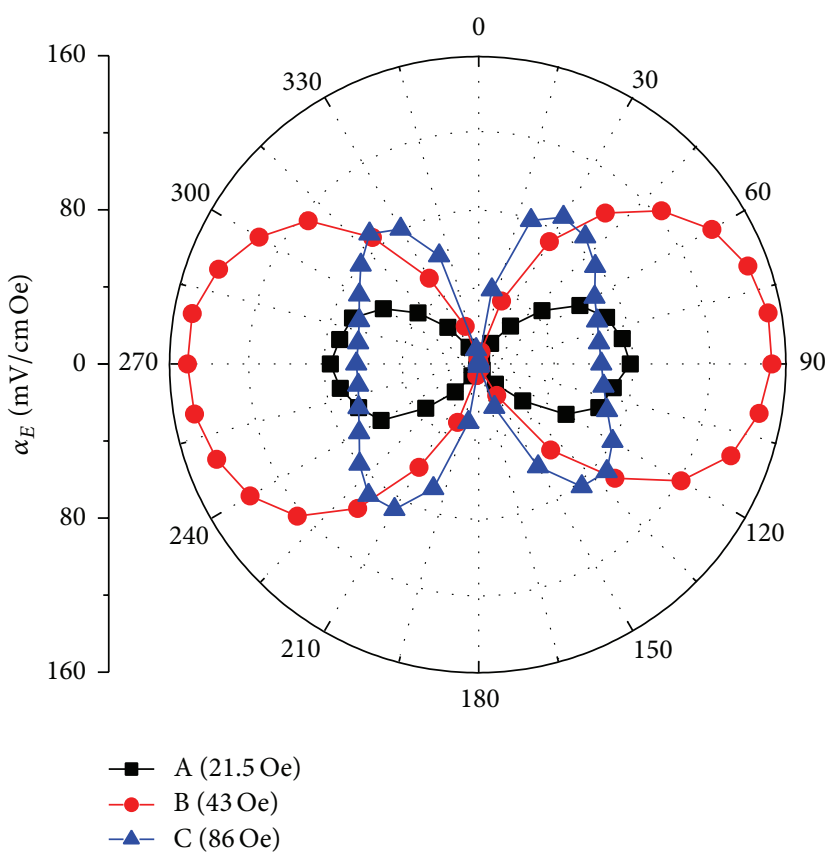

(b)

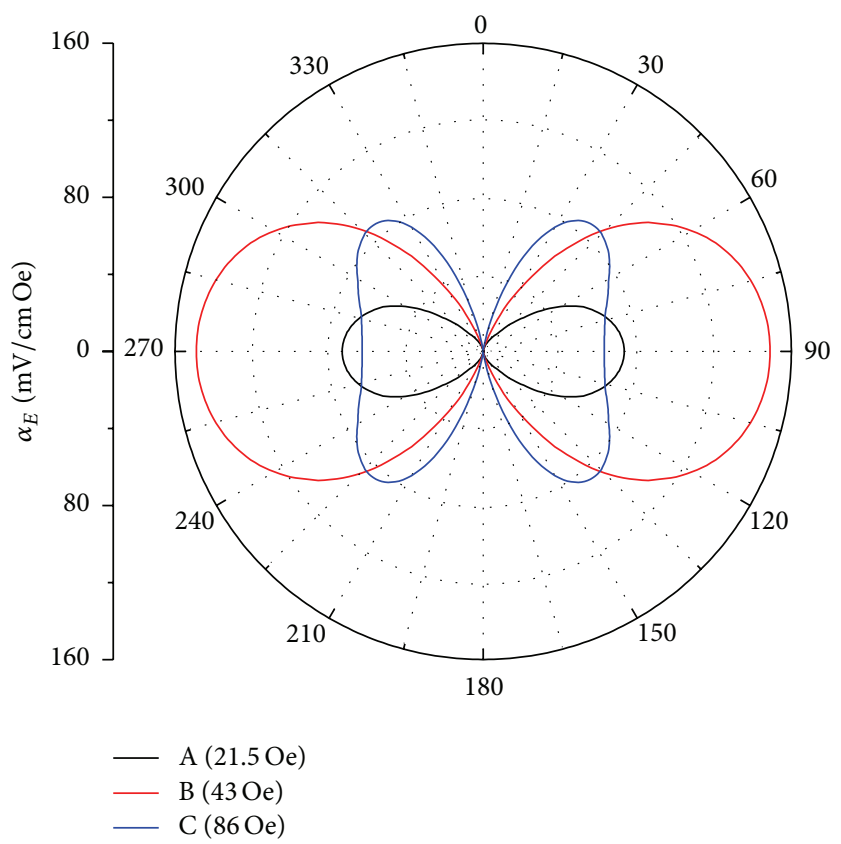

(c)

Figure 3: (a) Magnetic-field dependence of ME coefficient $\alpha_{E}$ in the FeBSiC/PZT/FeBSiC trilayer with a dimension of $10 \times 5 \times 0.5 \mathrm{~mm}^{3}$ for the PZT slab (Sample 2). (b) Experimentally measured polar plots of the angular dependent ME coefficient $\alpha_{E}$ in Sample 2 and (c) the computer simulation.

Thus, for the potential ME sensor applications, by simply presetting the $H_{\mathrm{dc}}$ bias-dependent ME voltage coefficient $\alpha_{E}$ at $\theta=90^{\circ}$ as the standard ME response, the ME outputs upon magnetic-fields with arbitrary amplitudes and orientations can in principle be obtained via computer simulations. When the ME sensor rotated in the magnetic-field, the magnetic-field can be decomposed along two directions: parallel and perpendicular to the surface plane. As the perpendicular direction almost has no contribution to the ME output, the ME output caused by the parallel direction can be obtained by the presetting standard ME response. By further comparing the magnetic-field-orientation dependent $\alpha_{E}$ measured in practical environment to the simulation results, both the direction and amplitude of the external 
magnetic-field can be conveniently identified. Following this procedure, we design a virtual ME sensor prototype using the Labview software based on the FeBSiC/PZT/FeBSiC trilayer, and the magnetic-field-orientation dependent $\alpha_{E}$ measured upon magnetic-fields of various amplitudes is numerically calculated as shown in Figure 2(c). As seen, the good match between experiments and simulations demonstrates the applicability of such ME sensors in detecting magneticfield with uncertain amplitudes and/or orientations.

Moreover, similar dependency of the magnetic-fieldorientation on the ME voltage coefficient $\alpha_{E}$ is obtained in the $\mathrm{FeBSiC} / \mathrm{PZT} / \mathrm{FeBSiC}$ trilayer with a dimension of $10 \times 5 \times$ $0.5 \mathrm{~mm}^{3}$ for the PZT slab (i.e., Sample 2), as shown in Figures $3(\mathrm{a})-3(\mathrm{c})$. The larger in-plane aspect ratio and the smaller volume of Sample 2 should be helpful in designing rod-shaped probe for practical sensor applications [19]. Moreover, the large ME voltage coefficient $\alpha_{E}$ in Sample $2(\sim 150 \mathrm{mV} / \mathrm{cmOe})$ guarantees a high magnetic-field sensitivity similar to that in Sample 1 with $\alpha_{E} \sim 138 \mathrm{mV} / \mathrm{cmOe}$.

\section{Conclusions}

In summary, the dependence of magnetic-field-orientation on the ME voltage coefficient $\alpha_{E}$ has been studied for $\mathrm{FeBSiC} / \mathrm{PZT} / \mathrm{FeBSiC}$ trilayers. Based on the experimentally measured magnetic-field dependent $\alpha_{E}$ with $H_{\mathrm{dc}}$ parallel to the surface of the PZT slab, the magnetic-field-orientation dependent ME voltage output upon magnetic-fields of various amplitudes can be obtained via computer simulations. The simulation results agree well with corresponding experimental measurements, demonstrating the applicability of such ME sensors in detecting magnetic-fields with uncertain amplitudes and/or orientations in environment.

\section{Conflict of Interests}

The authors declare that there is no conflict of interests regarding the publication of this paper.

\section{Acknowledgments}

This work was supported by Beijing Education Committee (Grant no. 20121000301) and the NSF of China (Grants nos. 11234005 and 51221291).

\section{References}

[1] H. Schmid, "Multi-ferroic magnetoelectrics," Ferroelectrics, vol. 162, no. 1, pp. 317-338, 1994.

[2] M. Fiebig, "Revival of the magnetoelectric effect," Journal of Physics D: Applied Physics, vol. 38, no. 8, pp. R123-R152, 2005.

[3] C.-W. Nan, M. I. Bichurin, S. Dong, D. Viehland, and G. Srinivasan, "Multiferroic magnetoelectric composites: historical perspective, status, and future directions," Journal of Applied Physics, vol. 103, no. 3, Article ID 031101, 2008.

[4] K. Mori and M. Wuttig, "Magnetoelectric coupling in TerfenolD/polyvinylidenedifluoride composites," Applied Physics Letters, vol. 81, no. 1, article 100, 2002.
[5] J. Ryu, S. Priya, A. Vázquez Carazo, K. Uchino, and H.-E. Kim, "Effect of the magnetostrictive layer on magnetoelectric properties in lead zirconate titanate/terfenol-D laminate composites," Journal of the American Ceramic Society, vol. 84, no. 3-12, pp. 2905-2908, 2001.

[6] S. Dong, J. Zhai, J. Li, and D. Viehland, "Near-ideal magnetoelectricity in high-permeability magnetostrictive/ piezofiber laminates with a (2-1) connectivity," Applied Physics Letters, vol. 89, no. 25, Article ID 252904, 2006.

[7] J. Robert, P. Andre, K. Reinhard, and Q. Eckhard, "Giant magnetoelectric effect in thin-film composites," Journal of the American Ceramic Society, vol. 96, no. 6, pp. 1673-1681, 2013.

[8] C. W. Nan, M. Li, and J. H. Huang, "Calculations of giant magnetoelectric effects in ferroic composites of rare-earthiron alloys and ferroelectric polymers," Physical Review BCondensed Matter and Materials Physics, vol. 63, no. 14, Article ID 144415, 2001.

[9] W. Eerenstein, N. D. Mathur, and J. F. Scott, "Multiferroic and magnetoelectric materials," Nature, vol. 442, no. 7104, pp. 759765, 2006.

[10] O. V. Antonenkov, M. I. Bichurin, D. A. Filippov, V. M. Petrov, and G. Srinivasan, "Electric-field-induced shift of the magnetic resonance line in ferrite-piezoelectric composites," Technical Physics Letters, vol. 31, no. 8, pp. 673-675, 2005.

[11] J. Zhai, Z. Xing, S. Dong, J. Li, and D. Viehland, "Detection of pico-Tesla magnetic fields using magneto-electric sensors at room temperature," Applied Physics Letters, vol. 88, no. 6, Article ID 062510, 2006.

[12] C.-S. Park, C.-W. Ahn, S.-C. Yang, and S. Priya, "Dimensionally gradient magnetoelectric bimorph structure exhibiting wide frequency and magnetic dc bias operating range," Journal of Applied Physics, vol. 106, no. 11, Article ID 114101, 2009.

[13] J. Das, J. Gao, Z. Xing, J. F. Li, and D. Viehland, “Enhancement in the field sensitivity of magnetoelectric laminate heterostructures," Applied Physics Letters, vol. 95, no. 9, Article ID 092501, 2009.

[14] Y. Wang, D. Gray, D. Berry et al., "An extremely low equivalent magnetic noise magnetoelectric sensor," Advanced Materials, vol. 23, no. 35, pp. 4111-4114, 2011.

[15] J. Y. Zhai, N. Cai, Z. Shi, Y. H. Lin, and C. W. Nan, "Coupled magnetodielectric properties of laminated $\mathrm{PbZrTiO} / \mathrm{NiFeO}$ ceramics," Journal of Applied Physics, vol. 95, no. 3, pp. 56855690, 2004.

[16] Y. Lin, N. Cai, J. Zhai, G. Liu, and C.-W. Nan, "Giant magnetoelectric effect in multiferroic laminated composites," Physical Review B-Condensed Matter and Materials Physics, vol. 72, no. 1, Article ID 012405, 2005.

[17] J. Zhai, Z. Xing, S. Dong, J. Li, and D. Viehland, "Magnetoelectric laminate composites: an overview," Journal of the American Ceramic Society, vol. 91, no. 2, pp. 351-358, 2008.

[18] J. Wang, L. Wang, G. Liu, Z. Shen, Y. Lin, and C. W. Nan, "Substrate effect on the magnetoelectric behavior of $\mathrm{Pb}\left(\mathrm{Zr}_{0.52} \mathrm{Ti}_{0.48}\right) \mathrm{O}_{3}$ Film-On-CoFe $\mathrm{O}_{4}$ bulk ceramic composites prepared by direct solution spin coating," Journal of the American Ceramic Society, vol. 92, no. 11, pp. 2654-2660, 2009.

[19] D. T. H. Giang and N. H. Duc, "Magnetoelectric sensor for microtesla magnetic-fields based on $\left(\mathrm{Fe}_{80} \mathrm{Co}_{20}\right)_{78} \mathrm{Si}_{12} \mathrm{~B}_{10} / \mathrm{PZT}$ laminates," Sensors and Actuators A: Physical, vol. 149, no. 2, pp. 229-232, 2009. 

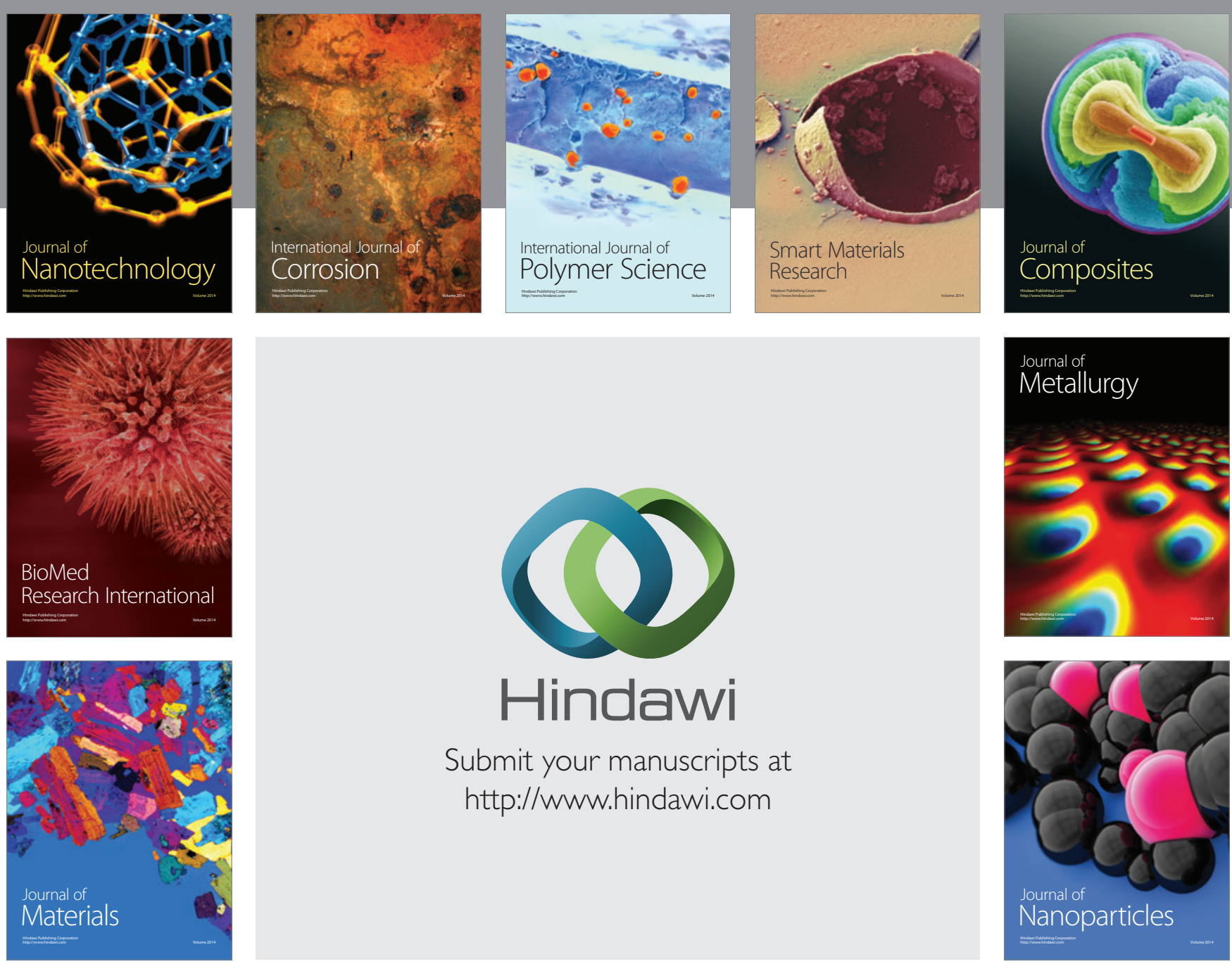

Submit your manuscripts at http://www.hindawi.com
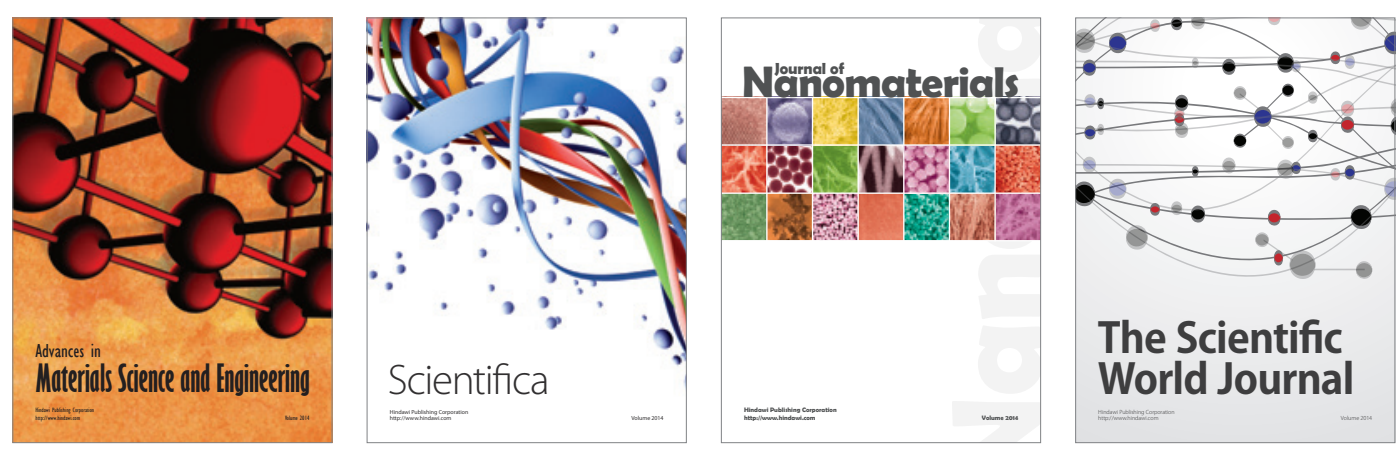

\section{The Scientific World Journal}
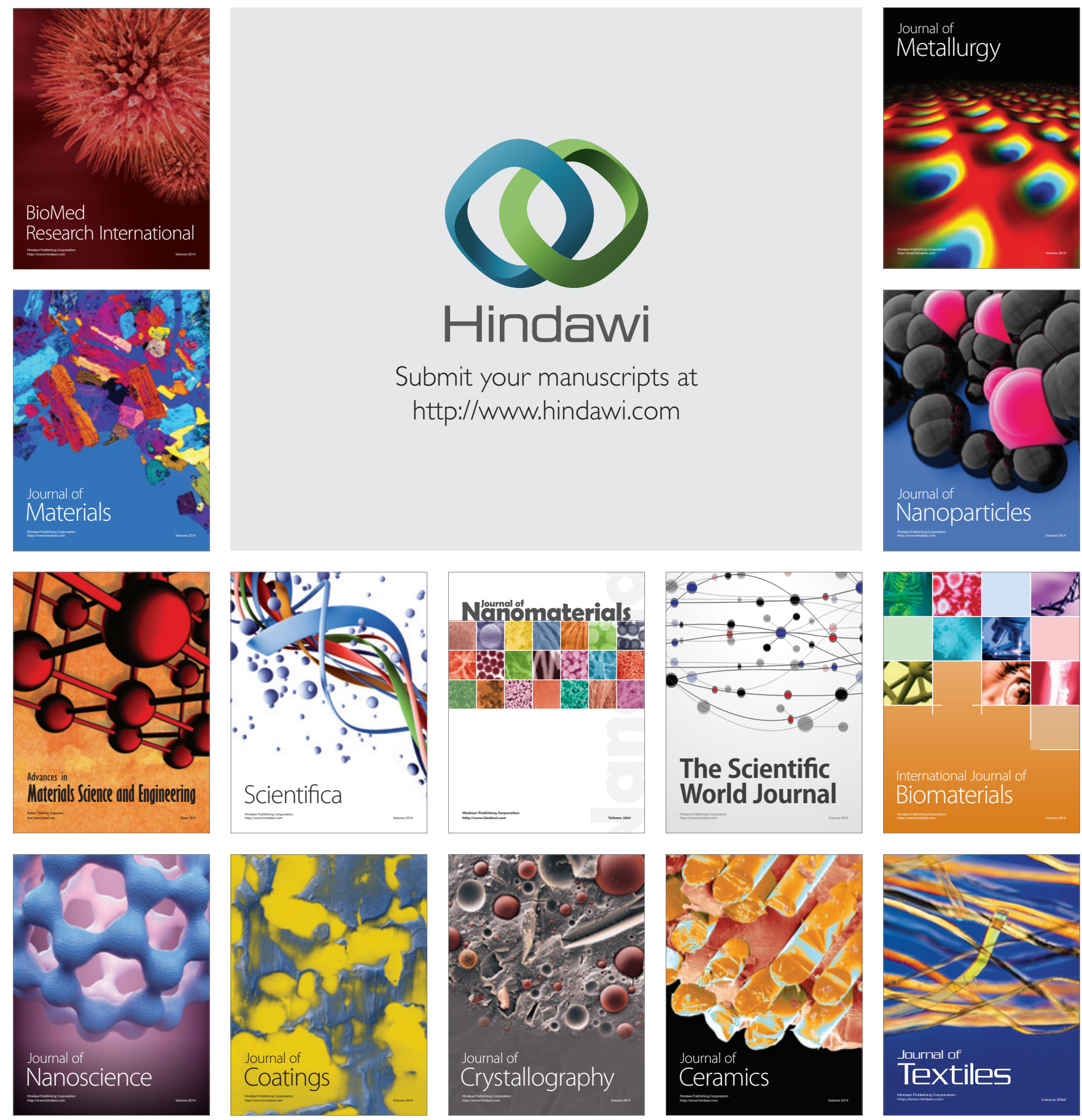